\title{
Influence of material choice on the force delivery of bimaxillary tooth positioners on canine malpositions
}

\section{Der Einfluss der Materialwahl auf die Kraftabgabe von bimaxillären Zahnpositionern bei Fehlstellungen der Eckzähne}

\author{
Didier Lochmatter, Markus Steineck, Lorenz Brauchli ${ }^{1}$
}

\begin{abstract}
Objective. Since their introduction in 1945 , tooth positioners have been used to treat a range of malpositions. Although the original appliance was made of natural rubber, today's tooth positioners are fabricated from various elastic, transparent materials. It was the aim of this study to evaluate the forces and moments produced by current positioners on various upper canine malpositions.

Material and methods. Seven positioners of different materials were tested on 11 upper canine malpositions: $0.25,0.5,1 \mathrm{~mm}$ supraposition; $0.25,0.5,1 \mathrm{~mm}$ infraposition; $5^{\circ}, 10^{\circ}, 20^{\circ}$ mesiorotation; $5^{\circ}$ mesioinclination, $5^{\circ}$ buccal root torque. We measured forces and moments in vitro after bite closure by $0.5 \mathrm{~mm}$, and opening by $1 \mathrm{~mm}$ using a three-dimensional robotic device. All measurements were taken in a temperature-controlled environment at $36^{\circ} \mathrm{C}$.

Results. The forces and moments measured at the canine varied greatly among the different positioners, with the thermoformed EVA positioner showing much greater forces and moments in almost all malpositions. At initial closure, we observed intrusive forces of 6-32 $\mathrm{N}$ for suprapositions, $0-11 \mathrm{~N}$ intrusive forces for infrapositions, 0-20 Nmm for mesiorotations, 6-12 Nmm for mesioinclinations, and $-23 \mathrm{Nmm}$ to $5 \mathrm{Nmm}$ for buccal root torque. All positioners were most effective on suprapositioned teeth. Very low or negligible correctional forces and moments in conjunction with all infrapositions and $5^{\circ}$ with rotation were noted. Labial root torque led to unpredictable moments.

Conclusion. Positioner material plays a major role in delivering forces to the teeth. However, tooth positioners did not induce corrective forces in all the malpositions tested. Clinically relevant correctional forces or moments in conjunction with all supraposi-
\end{abstract}

${ }^{1}$ Department of Orthodontics and Pedodontics, University of Basel, Basel, Switzerland

Received: January 13, 2011; accepted: November 21, 2011; published online: March 3, 2012

\section{Zusammenfassung}

Ziel. Seit ihrer Einführung 1945 sind Zahnpositioner zur Behandlung einer Reihe von Fehlstellungen eingesetzt worden. Die ursprüngliche Apparatur bestand aus Naturgummi; heute dagegen werden Zahnpositioner aus verschiedenen elastischen, transparenten Materialien hergestellt. Ziel der vorliegenden Arbeit war die Bewertung der Kräfte und Drehmomente, mit denen die derzeit verwendeten Positioner auf verschiedene Fehlstellungen eines oberen Eckzahns einwirken.

Material und Methodik. Sieben Positioner aus verschiedenen Materialien wurden an 11 Fehlstellungen eines oberen Eckzahns getestet: 0,25, 0,5, $1 \mathrm{~mm}$ Supraposition; 0,25, 0,5, $1 \mathrm{~mm}$ Infraposition; $5^{\circ}, 10^{\circ}, 20^{\circ}$ Mesiorotation; $5^{\circ}$ Mesioinklination, $5^{\circ}$ bukkaler Wurzeltorque. Die Kräfte und Drehmomente nach einer Schließung des Bisses um 0,5 mm und einer Öffnung um $1 \mathrm{~mm}$ wurden in einer In-vitro-Versuchsanordnung unter Verwendung einer dreidimensionalen robotisierten Vorrichtung getestet. Alle Messungen wurden in einer Umgebung mit einer kontrollierten Temperatur von $36^{\circ} \mathrm{C}$ vorgenommen.

Ergebnisse. Die am Eckzahn gemessenen Kräfte und Drehmomente wichen für die verschiedenen Positioner stark voneinander $a b$, wobei der thermogeformte EVA-Positioner für fast alle Fehlstellungen eine erheblich größere Kraft- und Momentabgabe zeigte. Beim initialen Kieferschluss wurden für Suprapositionen Intrusionskräfte von 6-32 N, für Infrapositionen Intrusionskräfte von $0-11 \mathrm{~N}$, für Mesioinklinationen $0-20 \mathrm{Nmm}$ und für bukkalen Wurzeltorque-23 bis $5 \mathrm{Nmm}$ gemessen. Alle Positioner zeigten sich bei Zähnen mit Suprapositionen am wirksamsten. Sehr geringe oder vernachlässigbare Kräfte/Drehmomente wurden bei allen Infrapositionen und der $5^{\circ}$ Rotation festgestellt. Beim labialen Wurzeltorque ergaben sich nicht vorhersagbare Drehmomente. Schlussfolgerung. Die für Positioner verwendeten Materialien spielen eine wichtige Rolle bei der Kraftabgabe an die Zähne. Jedoch wurden durch die Positioner nicht bei allen getesteten Fehlstellungen Korrekturkräfte induziert. Klinisch relevante Korrekturkräfte

J Orofac Orthop 2012; 73:104-115

DOI 10.1007/s00056-011-0067-7 
tions, rotations of $10^{\circ}$ and $20^{\circ}$ as well as mesial tipping of the canine were observed.

\author{
Keywords \\ Positioner · Material · Force · Moment · Canine
}

\section{Introduction}

Since introduction of the elastic positioner in 1945 [8], this appliance has been used for manifold purposes ranging from pure retention to active tooth movement. The original tooth positioner was made of natural rubber [8], which was replaced by a variety of materials including thermoelastic acryl, polyether urethane, ethylene vinyl acetate, and silicone $[3,11,21]$. The tooth positioner was developed in 1945 to provide "a simple appliance that would influence all of the teeth to flow into their best possible position" [8]. It was intended as a finishing appliance that would allow for earlier debonding of the fixed orthodontic appliance, and as a retention appliance. However, it soon became apparent that the positioner was not an ideal retention device $[3,5,20]$. Instead it was recommended as a settling device for several weeks. In comparison with conventional retainers, more tooth contacts were reported with the positioner after 3 months of wear [22]. However, another report found no significant improvement in tooth contact through positioner wear [21].

The tipping of teeth within reasonable limits of $1-2 \mathrm{~mm}$ is frequently described as the most relevant tooth movement during positioner therapy $[3,6,10,21]$. Rotational correction is advantageous in incisors but difficult in all other teeth [4]. Finally, extrusive and intrusive movements are believed to be unpredictable [6, 21]. In addition to moving individual teeth, the positioner has also been advocated for improving the centric condylar position. There is evidence that centric occlusion improved significantly during the first 2 weeks of continuous wear [13]. An investigation of how the fabrication method of tooth positioners influences centric condylar occlusion revealed that positioners made with a facebow registration and a centric interocclusal registration were more efficient than those without individual registrations [23]. The retention of intermaxillary relationships such as overjet and overbite in Class II patients and those with deep or open bite have also been described [5, 7, 8, 22].

The aim of this study was to investigate the forces and moments produced by different positioner materials on canine malpositions.

\section{Material and methods}

The effect of seven widely used positioner materials were investigated in this study: polyether urethane [shore hardness (sh) 70: Professional Positioners Inc., Racine, WI, USA], ethylene vinyl acetate (sh 75: Glenroe Technologies, Bradenton, FL, USA; sh 80: Scheu Dental GmbH, Iserlohn, Germany), and oder Drehmomente fanden sich für alle Suprapositionen, Rotationen von $10^{\circ}$ und $20^{\circ}$ sowie für Mesialkippungen des Eckzahns.

\section{Schlüsselwörter}

Positioner · Material · Kraft · Drehmoment · Eckzahn

\section{Einleitung}

Seit der Einführung des elastischen Positioners im Jahr 1945 [8] wurde diese Apparatur für eine Vielzahl von therapeutischen Zwecken von der reinen Retention bis zur aktiven Zahnbewegung verwendet. Der ursprüngliche Zahnpositioner bestand aus Naturgummi [8], das durch eine Vielzahl von Materialien einschließlich thermoelastisches Acryl, Polyetherurethan, Ethylenvinyl-Acetat und Silikon ersetzt wurde [3, 11, 21]. Die Entwicklung des Zahnpositioners im Jahre 1945 war von der Absicht getragen, „eine einfache Apparatur zu finden, die so auf alle Zähne einwirken würde, dass sie sich fließend in ihre bestmögliche Position bewegen" [8]. Gedacht war er als Apparatur für die Schlussphase der Behandlung, die ein früheres Debonding der festsitzenden kieferorthopädischen Apparatur ermöglichen sollte, sowie als Retentionsgerät. Schnell wurde jedoch bemerkt, dass der Positioner keine ideale Retentionsapparatur war $[3,5,20]$. Stattdessen wurde er zur Behandlungskonsolidierung im Rahmen eines mehrwöchigen Einsatzes empfohlen. Im Vergleich zu konventionellen Retainern fanden sich beim Positioner mehr Zahnkontakte nach dreimonatigem Tragen [22]. In einem anderen Bericht jedoch wurde keine signifikante Verbesserung des Zahnkontakts durch das Tragen des Positioners festgestellt [21].

Eine Zahnkippung innerhalb vernünftiger Grenzen von 1-2 $\mathrm{mm}$ wird oft als die relevanteste Art der Zahnbewegung während der Therapie mit dem Positioner beschrieben [3, 6, 10, 21]. Die Durchführung von Rotationskorrekturen sind bei den Frontzähnen günstig, aber für alle anderen Zähne schwierig [4]. Schließlich werden Extrusions- und Intrusionsbewegungen als nicht vorhersagbar bezeichnet $[6,21]$. Neben der Bewegung einzelner Zähne ist die Verwendung eines Positioners auch für eine verbesserte Zentrierung der Kondylenposition befürwortet worden. Nach den ersten zwei Wochen durchgehenden Tragens wurde eine signifikante Verbesserung der zentrischen Okklusion festgestellt [13]. Eine Untersuchung des Einflusses der Herstellungsmethode von Zahnpositionern auf die zentrische Kondylenposition in Okklusion ergab, dass mit Gesichtsbogenregistrierung und zentrischem interokklusalen Registrat hergestellte Positioner eine günstigere Wirkung zeigten als diejenigen ohne individuelle Registrate [23]. Schließlich wurde die Retention der intermaxillären Beziehungen wie etwa Overjet und Overbite bei Klasse-II-Patienten sowie bei Patienten mit Tiefbiss oder offenem Biss beschrieben [5, 7, 8, 22].

Ziel der vorliegenden Studie war die Bewertung der Kräfte und Drehmomente, mit denen die derzeit verwendeten Positioner auf verschiedene Fehlstellungen der Eckzähne einwirken. 


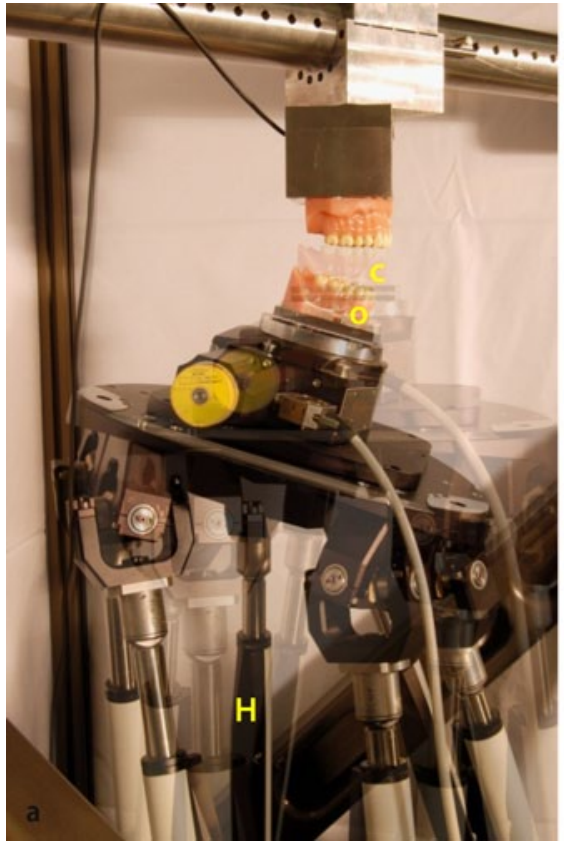

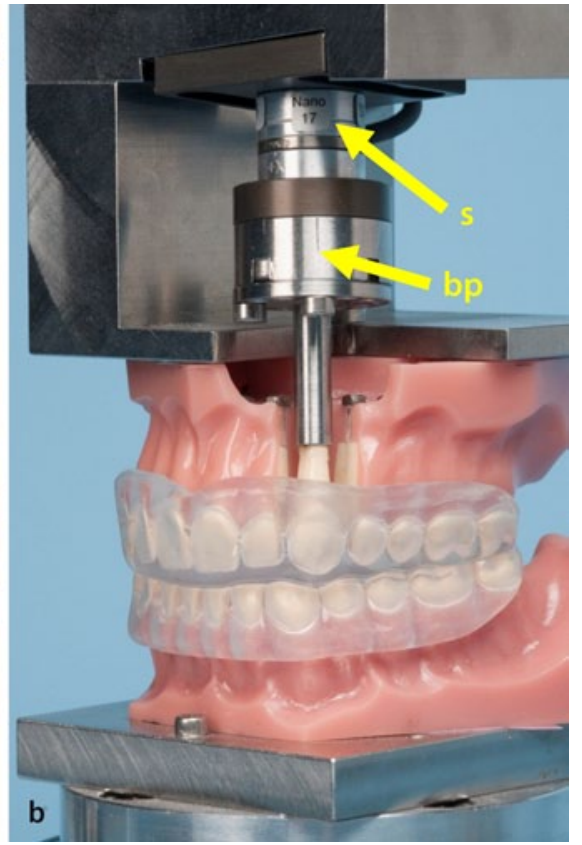

Figure 1. Experimental setup using a Hexapod $(\mathrm{H})$ for three-dimensional in vitro simulation of positioner therapy. a Supraposition of the Hexapod with the Frasaco models in the end-position of mouth opening $(0)$ and in an almost closed position (C). b Detail of the positioner setup: various canine malpositions were simulated by exchanging the base plates $(b p)$ interposed between the six-axis sensor $(s)$ and tooth

Abbildung 1. Versuchsanordnung mit einem Hexapod (H) für die dreidimensionale In-vitro-Simulation einer Positioner-Therapie. a Supraposition des Hexapod über den Frasaco-Modellen in der Endstellung der Mundöffnung $(O)$ und in fast geschlossener Stellung (C). b Detailaufnahme der Positioner-Anordnung: Durch Austausch der zwischen dem Sechs-Achsen-Sensor (s) und dem Zahn positionierten Basisplatten $(b p)$ wurden verschiedene Fehlstellungen der Eckzähne simuliert two high temperature silicones (Silicone sh 50/70: Dr. Hinz Dental, Herne, Germany; Odontosil sh 50/60: Dreve Dentamid GmbH, Unna, Germany). Anatomical dental models (upper jaw: A-3 T 139, lower jaw: A-3 T 228; Frasaco GmbH, Tettnang, Germany) were used to simulate both jaws. All positioners were made on replicas of one set of models. To allow the left upper canine the necessary degree of movement, it had to be removed from the model, and the adjacent teeth reduced in width (Figure $1 \mathrm{~b}$ ). The canine was connected to a stainless steel rod, which was again fixed to the force sensor through an intermediate base plate (Figure 1b). The whole upper jaw as well as the canine were then adjusted in the control position, with no tooth displacement. A silicone impression was taken and replicas of the model and canine mounted on the force sensor constructed. It was on these models that the tooth positioners were fabricated. The lower jaw was replicated with silicone impressions without any modifications. All positioners were conventionally formed by the same laboratory technician except the Scheu EVA 80, which was made by thermoforming and supplied by the company technician.

Forces and moments during bite closure were evaluated for 11 canine malpositions. Tooth 23 was measured in supraposition $(0.25 \mathrm{~mm}, 0.5 \mathrm{~mm}, 1 \mathrm{~mm})$, infraposition $(0.25 \mathrm{~mm}, 0.5 \mathrm{~mm}, 1$ $\mathrm{mm})$, mesial rotation $\left(5^{\circ}, 10^{\circ}, 20^{\circ}\right)$, palatal crown torque $\left(5^{\circ}\right)$, and mesial inclination $\left(5^{\circ}\right)$. Eleven CNC-milled base plates were made according to the 11 malpositions, which could be secured into position between the force sensor and canine (Figure $1 \mathrm{~b}$ ). Thus, it was possible to exactly reproduce the different malpositions.

As mandibular movement on opening and closing is not linear, we calculated a bite curve based on rotational and translatory jaw movements using anatomical measurements. These included the diameter of the rotational cylinder of the condyles,

\section{Material und Methodik}

Untersucht wurde die Wirkung von sieben weit verbreiteten Positioner-Materialien: Polyetherurethan [Shore-Härte (sh) 70; Professional Positioners., Racine, WI, USA], Ethylenvinyl-Acetat (sh 75: Glenroe Technologies, Bradenton, FL, USA; sh 80: Scheu Dental, Iserlohn, Deutschland) sowie zwei Hochtemperatur-Silikone (Silikon sh 50/70; Dr. Hinz Dental, Herne, Deutschland; Odontosil sh 50/60: Dreve Dentamid, Unna, Deutschland). Zur Simulation beider Kiefer wurden anatomische Zahnmodelle (Oberkiefer: A-3 T 139, Unterkiefer: A-3 T 228; Frasaco, Tettnang, Deutschland) verwendet. Alle Positioner wurden auf Replikaten eines Satzes von Modellen angefertigt. Um dem linken oberen Eckzahn genügend Bewegungsfreiheit zu lassen, musste er aus dem Modell herausgelöst und die Nachbarzähne von der Breite her reduziert werden (Abbildung 1b). Der Eckzahn wurde mit einem Edelstahlstab verbunden, der seinerseits über eine dazwischenliegende Basisplatte mit dem Kraftsensor verbunden war (Abbildung 1b). Der gesamte Oberkiefer sowie der Eckzahn wurden dann in die Kontrollposition ohne Zahnverschiebung gebracht. Es wurde ein Silikonabdruck genommen und Replikate des Modells und des auf dem Kraftsensor montierten Eckzahns angefertigt, Auf der Basis dieser Modelle wurden die Zahnpositioner hergestellt. Die Replikate des Unterkiefers wurden mit Hilfe von Silikonabdrücken ohne irgendwelche Modifikationen angefertigt. Alle Positioner wurden auf konventionelle Art und Weise durch denselben Zahntechniker hergestellt, mit Ausnahme des durch Thermoformung hergestellten Scheu EVA 80, der durch den Techniker des Unternehmens gefertigt wurde.

Die beim Zubiss auftretenden Kräfte und Drehmomente wurden für 11 Fehlstellungen des Eckzahns getestet. Zahn 23 wurde in Supraokklusion $(0,25 \mathrm{~mm}, 0,5 \mathrm{~mm}, 1 \mathrm{~mm})$, Infraok- 


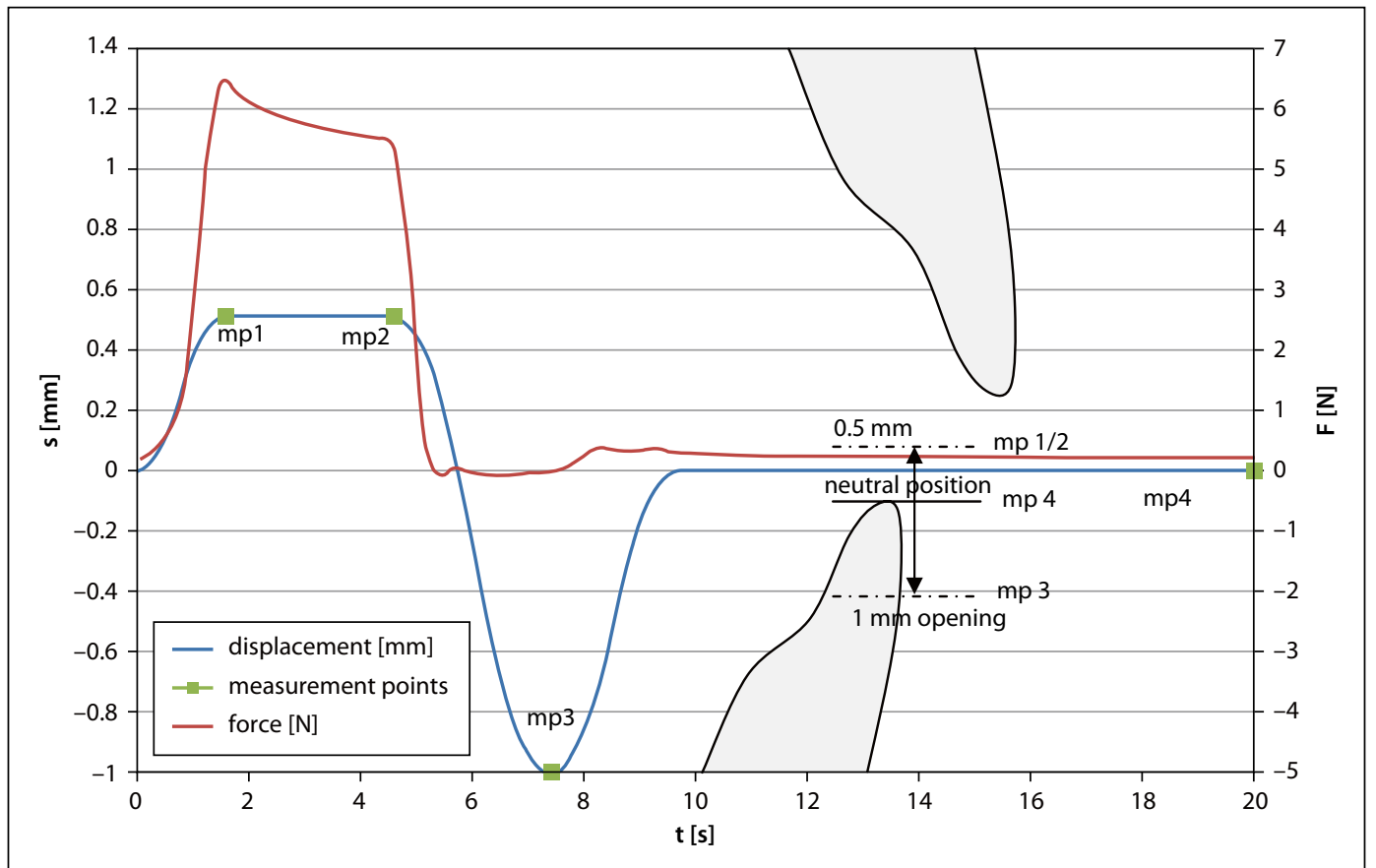

Figure 2. Typical experimental curve: the blue line shows one experimental cycle with all measurement points: $\mathrm{mp} 1 \mathrm{clo}-$ sure of $0.5 \mathrm{~mm}$ and immediate measurement, $\mathrm{mp} 2$ measurement after a $3 \mathrm{~s} \mathrm{lag} \mathrm{time} \mathrm{at} \mathrm{clo-}$ sure of $0.5 \mathrm{~mm}, \mathrm{mp} 3$ bite opening of $1 \mathrm{~mm}, \mathrm{mp} 4$ neutral position. The red line shows the development of a typical force in positioner testing

Abbildung 2. Eine typische Versuchskurve: Die blaue Linie zeigt einen Versuchszyklus mit allen Messpunkten: $\mathrm{mp} 1=\mathrm{Kie}-$ ferschluss von $0,5 \mathrm{~mm}$ und sofortiger Messung, $\mathrm{mp} 2=$ Messung mit zeitlicher Verzögerung und einem Kieferschluss von $0,5 \mathrm{~mm}, \mathrm{mp} 3=$ Bissöffnung von $1 \mathrm{~mm}, \mathrm{mp} 4=$ neutrale Stellung. Die rote Linie zeigt eine typische Kraftentwicklung beim Testen eines Positioners
Table 1. Specifications of the Hexapod Paros (MiCos GmbH, Eschbach Germany) and FTD Nano 17 force sensor (ATI, Apex, NC, USA)

Tabelle 1. Spezifikationen des Hexapod Paros (MiCos, Eschbach, Deutschland) und des FTD Nano 17-Kraftsensors (ATI, Apex, NC, USA)

\begin{tabular}{lllll}
\hline & Paros Hexapod & & \multicolumn{2}{l}{ FTD Nano 17} \\
\cline { 2 - 5 } & Range & Repeatability & Range & Error \\
\hline $\mathrm{x}$ & -174 to $172 \mathrm{~mm}$ & $0.0076 \mathrm{~mm}$ & $25 \mathrm{~N}$ & $<1 \%$ \\
$\mathrm{y}$ & -170 to $170 \mathrm{~mm}$ & $0.0069 \mathrm{~mm}$ & $25 \mathrm{~N}$ & $<1 \%$ \\
$\mathrm{z}$ & 525 to $626 \mathrm{~mm}$ & $0.0006 \mathrm{~mm}$ & $25 \mathrm{~N}$ & $<1 \%$ \\
Rx/Mx & -20 to $20^{\circ}$ & $0.009^{\circ}$ & $0.25 \mathrm{Nm}$ & $<1 \%$ \\
Ry/My & -20 to $20^{\circ}$ & $0.008^{\circ}$ & $0.25 \mathrm{Nm}$ & $<1 \%$ \\
Rz/Mz & -40 to $40^{\circ}$ & $0.051^{\circ}$ & $0.25 \mathrm{Nm}$ & $<1 \%$ \\
\hline
\end{tabular}

the distance between the center of the condyles and mandibular incisors, as well as the normal opening-closing pattern as represented by the Posselt diagram [14]. To carry out the three-dimensional movement, a robotic device (Hexapod Paros; MiCos Gm$\mathrm{bH}$, Eschbach, Germany) in combination with a 6-axis force-moment sensor (FTD Nano 17; ATI, Apex, NC, USA) was used. Specifications of the Hexapod and force sensor are listed in Table 1. The Hexapod and a detail of the positioner setup are shown in Figure 1a, b. The Hexapod was placed within a temperature test chamber heated to $36^{\circ} \mathrm{C}\left( \pm 0.5^{\circ} \mathrm{C}\right)$. Each biting cycle lasted $20 \mathrm{~s}$. The measurements were taken sequentially for $60 \mathrm{~min}$, resulting in 180 biting cycles for each positioner, malposition, and measurement point $(\mathrm{mp})$. The jaw movement and four measurement points are illustrated in Figure 2. From the starting position, the jaws were closed by $0.5 \mathrm{~mm}$ on the calculated bite curve (duration $1.5 \mathrm{~s}$, resulting in $\mathrm{mp} 1$ ). This position was held for $3 \mathrm{~s}$ (resulting in klusion $(0,25 \mathrm{~mm}, 0,5 \mathrm{~mm}, 1 \mathrm{~mm})$, Mesiorotation $\left(5^{\circ}, 10^{\circ}, 20^{\circ}\right)$, mit palatalem Kronentorque $\left(5^{\circ}\right)$ und mesialer Kippung $\left(5^{\circ}\right)$ gemessen. Entsprechend den 11 Fehlstellungen wurden 11 CNCgefräste Basisplatten angefertigt, die zwischen dem Kraftsensor und dem Eckzahn in Position gebracht werden konnten (Abbildung 1b). So konnten die verschiedenen Fehlstellungen exakt reproduziert werden.

Die Unterkieferbewegung der Mandibula beim Öffnen und Schließen ist nicht linear. Deswegen wurde anhand der Drehund Translationsbewegungen des Kiefers unter Verwendung von anatomischen Messdaten eine Beißkurve erstellt. $\mathrm{Zu}$ diesen Messdaten gehörten der Durchmesser des Rotationszylinders der Kondylen, die Entfernung zwischen den Kondylenmittelpunkten und den Schneidezähnen des Unterkiefers, sowie das normale Öffnungs- und Schließmuster wie im Posselt-Diagramm dargestellt [14]. Zur Ausführung der dreidimensionalen Bewegung wurde eine robotisierte Vorrichtung (Hexapod Paros; MiCos, Eschbach, Deutschland) in Verbindung mit einem 6-Achsen-Kraft-/Bewegungssensor (FTD Nano 17; ATI, Apex, NC, USA) verwendet. Die Spezifikationen des Hexapod und des Kraftsensors sind in Tabelle 1 aufgelistet. Eine Darstellung des Hexapod sowie eine Detailaufnahme der Positioner-Anordnung finden sich in Abbildung 1a, b. Der Hexapod wurde in eine auf $36^{\circ} \mathrm{C}\left( \pm 0.5^{\circ}\right)$ temperierte Prüfkammer platziert. Jeder Beißzyklus hatte eine Dauer von 20 Sekunden. Die Messungen wurden sequenziell für 60 Minuten durchgeführt; das ergab 180 Beißzyklen pro Positioner, Fehlstellung und Messpunkt (mp). Die Kieferbewegung und die vier Messpunkte sind in Abbildung 2 dargestellt. Von der Ausgangsposition aus wurden die Kiefer von der berechneten Beißkurve aus um 0,5 mm geschlossen (Dauer 1,5 s, ergibt mp1). Diese Position wurde 3 Sekunden gehalten (ergibt mp2). Die Kiefer wurden dann um 1,5 mm ge- 
Table 2. Means and standard deviations of all correcting forces (in $\mathrm{N}$ ) at $\mathrm{mp} 1$ for supraposition and infraposition of tooth 23

Tabelle 2. Mittelwerte und Standardabweichungen aller (in N ausgedrückten) Korrekturkräfte in mp1 für Supraposition und Infraposition von Zahn Nr. 23

\begin{tabular}{|c|c|c|c|c|c|c|}
\hline \multirow[t]{2}{*}{ mp 1} & \multicolumn{3}{|c|}{ Supraposition } & \multicolumn{3}{|l|}{ Infraposition } \\
\hline & $0.25 \mathrm{~mm}$ & $0.5 \mathrm{~mm}$ & $1 \mathrm{~mm}$ & $0.25 \mathrm{~mm}$ & $0.5 \mathrm{~mm}$ & $1 \mathrm{~mm}$ \\
\hline EVA sh 75 & $17.87(0.15)$ & $19.61(0.09)$ & $32.00(0.00)$ & $2.63(0.03)^{d}$ & $2.02(0.03)^{a}$ & $0.26(0.01)^{a}$ \\
\hline Scheu- EVA sh 80 & $31.97(0.00)$ & - & - & $10.61(0.15)$ & $4.22(0.07)$ & $1.81(0.04)$ \\
\hline Odontosil sh 50 & $9.30(0.17)$ & $13.57(0.09)$ & $19.18(0.03)$ & $2.90(0.1)$ & $1.36(0.07)^{b}$ & $0.47(0.01)^{b}$ \\
\hline Odontosil sh 60 & $12.17(0.11)$ & $29.81(0.05)$ & - & $2.35(0.04)$ & $1.99(0.02)^{a}$ & $0.34(0.01)$ \\
\hline Silicone sh 50 & $6.12(0.04)$ & $12.75(0.03)$ & $20.14(0.03)$ & $1.86(0.04)$ & $1.10(0.04)^{c}$ & $0.07(0.00)$ \\
\hline Silicone sh 70 & $11.68(0.03)$ & $21.27(0.04)$ & $31.79(0.00)$ & $2.73(0.06)^{a}$ & $1.47(0.23)^{b}$ & $0.28(0.02)^{\mathrm{a}}$ \\
\hline Polyurethan sh 70 & $10.19(0.11)$ & $5.68(0.01)$ & $30.46(0.03)$ & $2.74(0.07)^{a}$ & $1.04(0.04)^{c}$ & $0.46(0.03)^{b}$ \\
\hline \multirow[t]{2}{*}{$\mathrm{mp} 2$} & \multicolumn{3}{|c|}{ Supraposition } & \multicolumn{3}{|l|}{ Infraposition } \\
\hline & $0.25 \mathrm{~mm}$ & $0.5 \mathrm{~mm}$ & $1 \mathrm{~mm}$ & $0.25 \mathrm{~mm}$ & $0.5 \mathrm{~mm}$ & $1 \mathrm{~mm}$ \\
\hline EVA sh 75 & $15.61(0.15)$ & $18.54(0.09)$ & $32.00(0.00)$ & $2.04(0.03)$ & $1.52(0.03)$ & $0.19(0.01)$ \\
\hline Scheu- EVA sh 80 & $31.34(0.21)$ & - & - & $8.01(0.14)$ & $3.18(0.08)$ & $1.39(0.03)$ \\
\hline Odontosil sh 50 & $8.85(0.19)$ & $13.17(0.10)$ & $18.75(0.06)$ & $2.77(0.11)$ & $1.30(0.07)^{a}$ & $0.43(0.01)^{a}$ \\
\hline Odontosil sh 60 & $11.78(0.14)$ & $29.38(0.09)$ & - & $2.29(0.04)$ & $1.92(0.03)$ & $0.33(0.01)$ \\
\hline Silicone sh 50 & $5.75(0.06)$ & $12.21(0.08)$ & $20.04(0.05)$ & $1.61(0.04)$ & $0.94(0.03)$ & $0.05(0.01)$ \\
\hline Silicone sh 70 & $11.18(0.09)$ & $20.57(0.09)$ & $31.78(0.00)$ & $2.48(0.07)^{a}$ & $1.34(0.06)^{\mathrm{a}, \mathrm{b}}$ & $0.25(0.01)$ \\
\hline Polyurethan sh 70 & $9.87(0.12)$ & $5.58(0.03)$ & $29.99(0.09)$ & $2.56(0.07)^{a}$ & $0.94(0.03)^{b}$ & $0.43(0.02)^{a}$ \\
\hline \multirow[t]{2}{*}{$\mathrm{mp} 3$} & \multicolumn{3}{|c|}{ Supraposition } & \multicolumn{3}{|l|}{ Infraposition } \\
\hline & $0.25 \mathrm{~mm}$ & $0.5 \mathrm{~mm}$ & $1 \mathrm{~mm}$ & $0.25 \mathrm{~mm}$ & $0.5 \mathrm{~mm}$ & $1 \mathrm{~mm}$ \\
\hline EVA sh 75 & $0.27(0.02)$ & $0.02(0.01)$ & $0.32(0.03)^{a}$ & $0.04(0.01)$ & $0.08(0.01)$ & $0.03(0.01)$ \\
\hline Scheu-EVA sh 80 & $0.01(0.00)$ & - & - & $0.01(0.01)$ & $0.09(0.01)$ & $0.05(0.01)$ \\
\hline Odontosil sh 50 & $0.33(0.01)^{\mathrm{a}}$ & $0.68(0.01)$ & $0.67(0.02)$ & $0.05(0.01)$ & $0.02(0.01)$ & $0.11(0.01)$ \\
\hline Odontosil sh 60 & $0.18(0.02)$ & $0.21(0.02)$ & - & $0.03(0.01)^{b}$ & $0.07(0.01)$ & $0.04(0.01)^{\mathrm{a}}$ \\
\hline Silicone sh 50 & $0.52(0.01)$ & $0.39(0.03)$ & $0.34(0.04)^{a}$ & $0.33(0.01)^{\mathrm{a}}$ & $0.05(0.01)$ & $0.05(0.01)$ \\
\hline Silicone sh 70 & $0.34(0.02)^{a}$ & $0.16(0.03)$ & $0.62(0.06)$ & $0.31(0.01)^{\mathrm{a}}$ & $0.01(0.01)^{a}$ & $0.02(0.00)^{\mathrm{a}}$ \\
\hline Polyurethan sh 70 & $0.06(0.01)$ & $0.08(0.01)$ & $1.71(0.05)$ & $0.03(0.01)^{b}$ & $0.00(0.01)^{a}$ & $0.00(0.00)$ \\
\hline \multirow[t]{2}{*}{$\mathrm{mp} 4$} & \multicolumn{3}{|c|}{ Supraposition } & \multicolumn{3}{|l|}{ Infraposition } \\
\hline & $0.25 \mathrm{~mm}$ & $0.5 \mathrm{~mm}$ & $1 \mathrm{~mm}$ & $-0.25 \mathrm{~mm}$ & $-0.5 \mathrm{~mm}$ & $1 \mathrm{~mm}$ \\
\hline EVA sh 75 & $0.77(0.02)$ & $1.87(0.05)$ & $13.93(0.30)$ & $0.12(0.01)$ & $0.14(0.01)$ & $0.03(0.01)^{\mathrm{a}}$ \\
\hline Scheu- EVA sh 80 & $3.49(0.09)$ & - & - & $0.72(0.01)^{b}$ & $0.25(0.01)$ & $0.05(0.01)^{c}$ \\
\hline Odontosil sh 50 & $3.11(0.03)^{\mathrm{a}}$ & $3.24(0.02)$ & $8.17(0.02)$ & $1.03(0.03)^{a}$ & $0.46(0.03)^{a}$ & $0.12(0.01)$ \\
\hline Odontosil sh 60 & $2.10(0.02)$ & $3.98(0.05)$ & - & $0.68(0.02)$ & $0.57(0.01)$ & $0.07(0.00)^{b}$ \\
\hline Silicone sh 50 & $1.88(0.03)$ & $3.16(0.01)$ & $8.40(0.02)$ & $0.88(0.02)$ & $0.43(0.02)^{a}$ & $0.03(0.00)^{\mathrm{a}}$ \\
\hline Silicone sh 70 & $3.07(0.01)^{\mathrm{a}}$ & $4.77(0.02)$ & $14.42(0.06)$ & $1.05(0.01)^{a}$ & $0.69(0.03)$ & $0.08(0.01)^{b}$ \\
\hline Polyurethan sh 70 & $2.52(0.07)$ & $1.95(0.01)$ & $16.29(0.03)$ & $0.73(0.03)^{b}$ & $0.18(0.01)$ & $0.06(0.01)^{c}$ \\
\hline
\end{tabular}

$\mathrm{a}, \mathrm{b}, \mathrm{c}$ Non-significant differences between positioners of one group are indicated by the same letter. Forces exceeding the measurement range of the force sensor are indicated by -. ${ }^{\mathrm{d}}$ Forces or moments indicate contraproductive forces or moments opposing the intended correction of the malposition are printed in bold. 


\begin{tabular}{|c|c|c|c|c|c|}
\hline \multirow[t]{2}{*}{ mp 1} & \multicolumn{3}{|c|}{ Mesiorotation } & \multirow{2}{*}{$\begin{array}{l}\text { Mesioinclination } \\
5^{\circ}\end{array}$} & \multirow{2}{*}{$\begin{array}{l}\text { Buccal root torque } \\
5^{\circ}\end{array}$} \\
\hline & $5^{\circ}$ & $10^{\circ}$ & $20^{\circ}$ & & \\
\hline EVA sh 75 & $2.35(0.09)$ & $6.65(0.05)$ & - & $9.04(0.26)^{\mathrm{a}}$ & $3.10(0.21)$ \\
\hline Scheu- EVA sh 80 & $0.14(0.04)$ & $18.48(0.23)$ & - & $11.89(0.95)^{c}$ & $23.08(1.00)^{d}$ \\
\hline Odontosil sh 50 & $0.80(0.02)$ & $4.52(0.02)$ & $10.74(0.02)$ & $6.20(0.22)^{b}$ & $1.76(0.37)^{\mathrm{a}}$ \\
\hline Odontosil sh 60 & $1.49(0.02)$ & $9.94(0.12)$ & $19.78(0.07)$ & $11.31(0.23)^{c}$ & $5.15(0.21)$ \\
\hline Silicone sh 50 & $1.03(0.02)$ & $2.53(0.02)$ & $9.08(0.02)$ & - & $1.83(0.23)$ \\
\hline Silicone sh 70 & $2.07(0.04)$ & $5.43(0.04)$ & $14.42(0.06)$ & $8.58(0.27)^{\mathrm{a}}$ & - \\
\hline Polyurethan sh 70 & $1.82(0.02)$ & $6.40(0.04)$ & $18.62(0.1)$ & $5.92(0.20)^{b}$ & $1.28(0.21)^{\mathrm{a}}$ \\
\hline \multirow[t]{2}{*}{$\mathrm{mp} 2$} & \multicolumn{3}{|c|}{ Mesiorotation } & Mesioinclination & Buccal root torque \\
\hline & $5^{\circ}$ & $10^{\circ}$ & $20^{\circ}$ & $5^{\circ}$ & $5^{\circ}$ \\
\hline EVA sh 75 & $2.23(0.08)$ & $6.32(0.05)^{\mathrm{a}}$ & - & $9.11(0.27)^{\mathrm{a}}$ & $3.97(0.19)$ \\
\hline Scheu- EVA sh 80 & $0.09(0.04)$ & $17.97(0.03)$ & - & $9.37(0.41)$ & $13.16(0.71)$ \\
\hline Odontosil sh 50 & $0.72(0.02)$ & $4.43(0.02)$ & $10.66(0.03)$ & $6.45(0.25)$ & $2.18(0.34)$ \\
\hline Odontosil sh 60 & $1.49(0.02)$ & $9.78(0.11)$ & $19.66(0.07)$ & $11.24(0.22)$ & $5.20(0.23)$ \\
\hline Silicone sh 50 & $1.02(0.02)$ & $2.41(0.02)$ & $8.98(0.03)$ & - & $2.00(0.24)$ \\
\hline Silicone sh 70 & $1.96(0.03)$ & $5.30(0.05)$ & $14.40(0.06)$ & $8.75(0.23)$ & - \\
\hline Polyurethan sh 70 & $1.71(0.03)$ & $6.34(0.04) a$ & $18.77(0.10)$ & $5.89(0.24)$ & $1.26(0.22)$ \\
\hline \multirow[t]{2}{*}{$\mathrm{mp} 3$} & \multicolumn{3}{|c|}{ Mesiorotation } & Mesioinclination & Buccal root torque \\
\hline & $5^{\circ}$ & $10^{\circ}$ & $20^{\circ}$ & $5^{\circ}$ & $5^{\circ}$ \\
\hline EVA sh 75 & $0.08(0.02)^{\mathrm{a}}$ & $1.56(0.02)$ & - & $8.99(0.36)^{\mathrm{a}}$ & $8.21(0.27)$ \\
\hline Scheu- EVA sh 80 & $0.18(0.02)$ & $0.07(0.03)^{a}$ & - & $7.69(0.30)$ & $1.73(0.24)$ \\
\hline Odontosil sh 50 & $0.01(0.02)^{b}$ & $0.01(0.02)$ & $3.03(0.02)$ & $4.18(0.29)$ & $3.59(0.33)$ \\
\hline Odontosil sh 60 & $0.92(0.03)$ & $3.60(0.11)$ & $11.72(0.09)$ & $6.53(0.26)$ & $4.87(0.24)$ \\
\hline Silicone sh 50 & $0.00(0.02)^{b}$ & $0.03(0.02)^{\mathrm{a}}$ & $3.29(0.05)$ & - & $5.08(0.34)$ \\
\hline Silicone sh 70 & $0.09(0.02)^{\mathrm{a}}$ & $0.56(0.04)$ & $7.24(0.08)$ & $9.53(0.27)^{\mathrm{a}}$ & - \\
\hline Polyurethan sh 70 & $0.06(0.02)$ & $0.18(0.02)$ & $0.48(0.02)$ & $1.83(0.29)$ & $0.60(0.33)$ \\
\hline \multirow[t]{2}{*}{$\mathrm{mp} 4$} & \multicolumn{3}{|c|}{ Mesiorotation } & Mesioinclination & Buccal root torque \\
\hline & $5^{\circ}$ & $10^{\circ}$ & $20^{\circ}$ & $5^{\circ}$ & $5^{\circ}$ \\
\hline EVA sh 75 & $0.13(0.04)$ & $0.53(0.02)$ & $0.00(0.02)$ & $9.32(0.27)^{\mathrm{a}}$ & $6.46(0.29)$ \\
\hline Scheu- EVA sh 80 & $1.32(0.02)$ & $5.83(0.11)$ & - & $6.45(0.25)$ & $3.21(0.24)^{b}$ \\
\hline Odontosil sh 50 & $0.10(0.02)$ & $1.72(0.02)$ & $5.90(0.02)$ & $4.06(0.31)$ & $0.82(0.30)^{\mathrm{a}}$ \\
\hline Odontosil sh 60 & $1.04(0.03)^{\mathrm{a}}$ & $4.21(0.10)$ & $13.12(0.06)$ & $8.21(0.23)$ & $3.55(0.25)^{b}$ \\
\hline Silicone sh 50 & $0.49(0.02)$ & $0.81(0.02)$ & $5.09(0.03)$ & - & $1.89(0.27)$ \\
\hline Silicone sh 70 & $1.04(0.03)^{\mathrm{a}}$ & $2.71(0.02)$ & $9.30(0.05)$ & $9.11(0.27)^{\mathrm{a}}$ & $10.82(0.28)$ \\
\hline Polyurethan sh 70 & $0.72(0.02)$ & $2.82(0.03)$ & $9.93(0.06)$ & $2.10(0.28)$ & $1.40(0.26)^{\mathrm{a}}$ \\
\hline
\end{tabular}




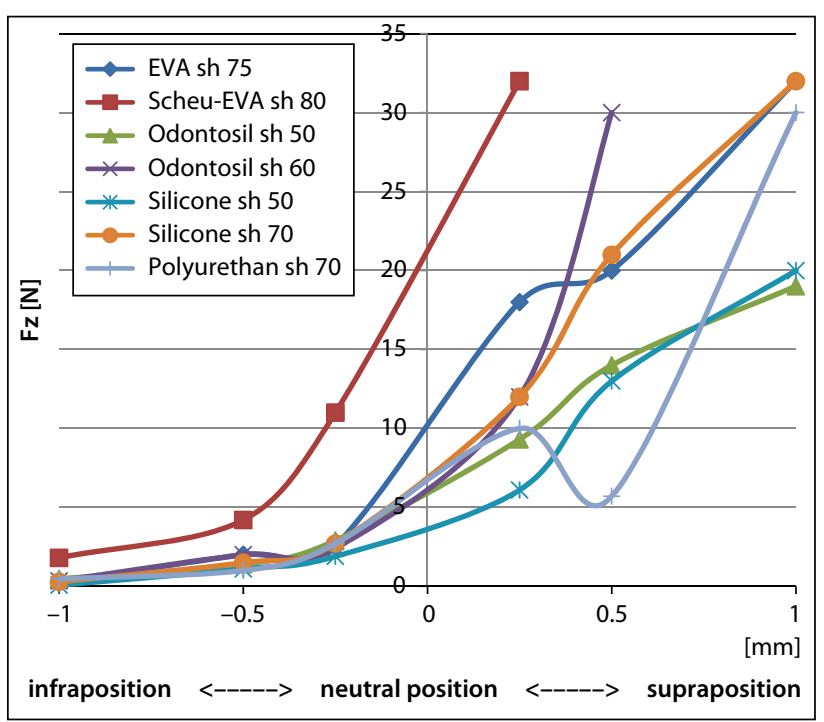

Figure 3. Graphic of force levels of vertical malpositions (infraposition and supraposition) of all positioners at measurement point 1 ( $0.5 \mathrm{~mm}$ bite closure). Strong intrusive forces were recorded for suprapositioned teeth. Although lighter, still intrusive forces were measured in conjunction with infrapositioned teeth

Abbildung 3. Graphische Darstellung von Kraftniveaus bei vertikalen Fehlstellungen (Infraposition und Supraposition) für alle Positioner in Messpunkt $1(0,5$ $\mathrm{mm}$ Kieferschluss). Für Zähne in Supraposition wurden starke Intrusionskräfte erfasst. Für Zähne in Infraposition wurden ebenfalls - wenn auch leichtere - Intrusionskräfte gemessen

mp2). The jaws were then opened $1.5 \mathrm{~mm}$ (duration $3 \mathrm{~s}$, resulting in $\mathrm{mp} 3$ ) and finally closed in the starting position (duration $1.5 \mathrm{~s}$ ). The remaining time of the cycle was used as a resting phase. The last value before starting a new cycle represented as mp4 showed positioner activity with no biting force. Forces and/or moments were, thus, produced on all teeth; however, they were only registered on the canine in malposition.

All four measurement points were analyzed for the force or moment acting against the malpositioned canine. We analyzed 90 cycles from a 30 -min test period, starting at $25 \mathrm{~min}$ and ending at $55 \mathrm{~min}$, recording a mean value with standard deviation from the 90 cycles for each measurement point. A Shapiro-Wilk normality test was used to assess normal distribution. As not all measurements showed normal distribution, a non-parametric ANOVA (Kruskal-Wallis and Dunns post test) was calculated for comparison of the forces and moments in association with the different positioners at measurement points 1-4, respectively, using Prism analysis software (Prism; GraphPad, San Diego, CA, USA). The level of significance was set at $\mathrm{p} \leq 0.05$.

\section{Results}

The results at all measurement points are summarized in Table 2 for supraposition and infraposition, and in Table 3 for rotation, inclination, and torque. Values in bold indicate forces or moments counteracting the intended correction of the prevail-

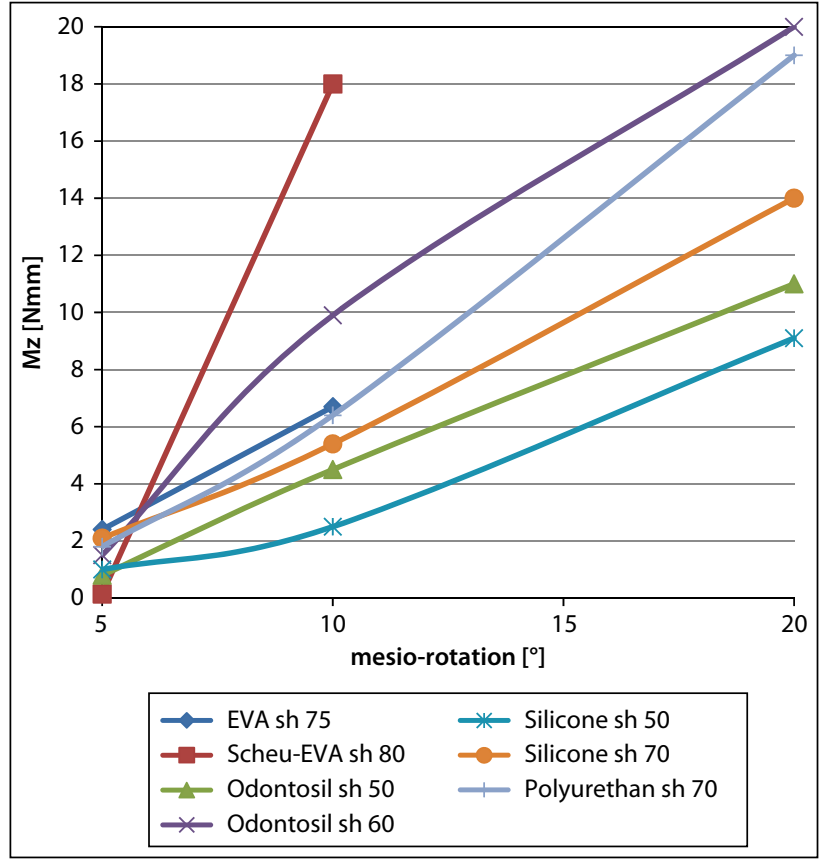

Figure 4. Graphic illustration of moments arising from different degrees of mesiorotation at measurement point $1\left(0.5 \mathrm{~mm}\right.$ bite closure). Small rotations of $5^{\circ}$ yielded nearly no corrective moments

Abbildung 4. Graphische Darstellung der bei verschiedenen Graden der Mesiorotation erzeugten Drehmomente in Messpunkt 1 (0,5 mm Kieferschluss). Für geringe Rotationen von $5^{\circ}$ wurden fast keine Korrekturdrehmomente erfasst

öffnet (Dauer 3 Sekunden, ergibt mp3) und schließlich in der Ausgangsposition geschlossen (Dauer 1,5 Sekunden). Die verbleibende Zeit des Zyklus diente als Ruhephase. Der letzte Wert vor Beginn eines neuen Zyklus, dargestellt als mp4, zeigte die Aktivität des Positioners ohne Wirken einer Beißkraft. Kräfte und Drehmomente wirkten somit auf alle Zähne ein, wurden jedoch nur bezüglich des Eckzahns mit der Fehlstellung erfasst.

Alle vier Messpunkte wurden hinsichtlich der auf den Eckzahn mit der Fehlstellung einwirkenden Kräfte und Drehmomente analysiert. Analysiert wurden 90 Zyklen aus einem Testzeitraum von 30 Minuten, beginnend bei 25 Minuten und endend bei 55 Minuten. Für alle Messpunkte wurden Mittelwerte mit einer Standardabweichung aus 90 Zyklen erfasst. Für die Bewertung der Normalverteilung wurde ein Shapiro-Wilk-Normalitätstest verwendet. Da nicht alle Messungen eine Normalverteilung aufwiesen, wurde ein nicht parametrischer ANOVA (nachträglicher Test nach Kruskal-Wallis und Dunns) für den Vergleich der Kräfte und Drehmomente für die verschiedenen Positioner an den Messpunkten 1 bis 4 berechnet, wobei jeweils die Analyse-Software Prism (Prism; GraphPad, San Diego, CA, USA) zum Einsatz kam. Das Signifikanzniveau wurde auf $\mathrm{p} \leq 0,05$ gesetzt.

\section{Ergebnisse}

Die Ergebnisse für alle Messpunkte wurden für Supraposition und Infraposition in Tabelle 2 und für Rotation, Inklination 


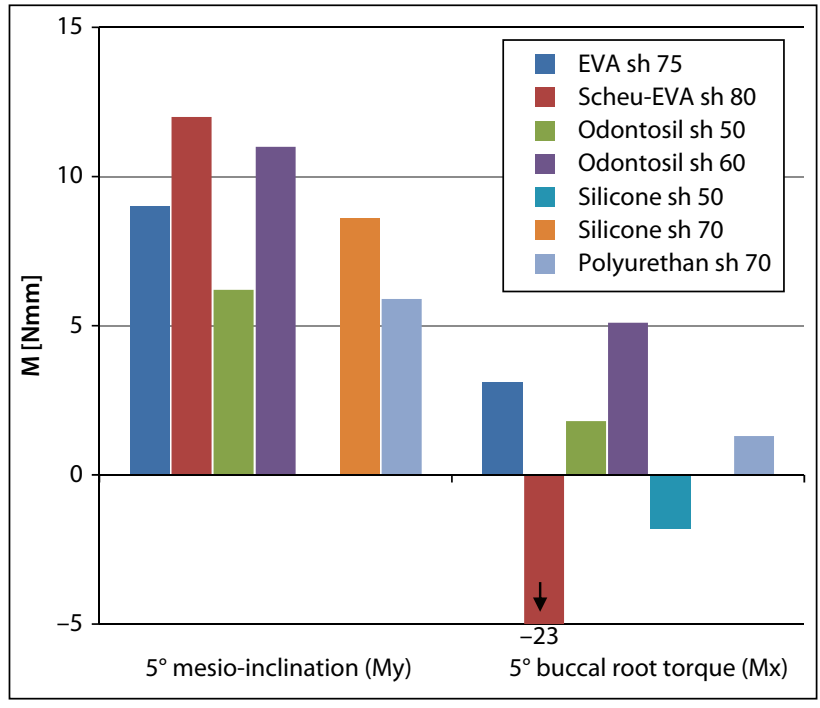

Figure 5. Graphic illustration of corrective moments of mesioinclined teeth and those with buccal root torque at measurement point 1 ( $0.5 \mathrm{~mm}$ bite closure). Positive values indicate beneficial therapeutic effects. Whereas the uprighting moments of tipped teeth may be clinically adequate, torque expression by the positioners appears both inadequate and unpredictable

Abbildung 5. Graphische Darstellung der Korrekturdrehmomente bei Zähnen mit Mesioinklination und einem bukkalen Wurzeltorque in Messpunkt $1(0,5$ $\mathrm{mm}$ Kieferschluss). Positive Werte verweisen auf einen nützlichen therapeutischen Effekt. Während die Aufrichtungsmomente von gekippten Zähnen in klinischer Hinsicht ausreichend sein mögen, scheint die Torque-Expression der Positioner nicht nur unzureichend, sondern auch nicht vorhersagbar

ing malposition. Almost all force levels from the seven positioners differed significantly from each other when the four measurement points were evaluated. Graphic illustrations of the forces and moments at mp 1 are provided in Figure 3, Figure 4 and Figure 5. All malpositions and all measurement points are summarized in a single illustration (Figure 6) for one exemplary positioner (Odontosil sh 50).

Canine supraposition led to considerable intrusive forces. At a supraposition of $0.25 \mathrm{~mm}$, most of the positioners' force level was approximately $10 \mathrm{~N}$, except for the thermoformed positioner, whose intrusive force measured $32 \mathrm{~N}$. At a supraposition of 0.5 $\mathrm{mm}$, most forces were approximately $20 \mathrm{~N}$, with the exception again being the thermoformed positioner, which yielded forces outside the measurement range of the sensor. At a supraposition of $1 \mathrm{~mm}$, the force levels reached $30 \mathrm{~N}$. However, the Odontosil sh 60 and Scheu-EVA D80 fell outside the measurement range.

Canine infraposition did not lead to obvious extrusive forces, but rather to less intrusive force levels as in the model with the canine in supraposition. At $0.25 \mathrm{~mm}$ of infraposition, all positioners showed intrusive forces of approximately $2.5 \mathrm{~N}$ with the exception of the thermoformed positioner which had higher forces. Even at $\mathrm{mp} 3$, only the two silicone positioners revealed a slight extrusive force of $0.3 \mathrm{~N}$. At $0.5 \mathrm{~mm}$ of infraposition, intrusive forces were reduced at the canine, and nearly no forces were detectable at $1 \mathrm{~mm}$ of infraposition. No level of infraposition led to extrusive forces. und Torque in Tabelle 3 zusammengefasst. Die halbfett gesetzten Werte stehen für der beabsichtigten Korrektur der vorherrschenden Fehlstellung entgegenwirkende Kräfte oder Drehmomente. Fast alle Kraftniveaus der sieben Positioner wiesen bei der Auswertung in Bezug auf die vier Messpunkte signifikante Unterschiede auf. Eine graphische Darstellung der Kräfte und Drehmomente in mp1 findet sich in Abbildung 3, Abbildung 4 und Abbildung 5. In Abbildung 6 sind alle Fehlstellungen sowie alle Messpunkte in einer Darstellung für einen beispielhaften Positioner zusammengefasst (Odontosil sh 50).

Die Supraposition des Eckzahnes führte zur Erzeugung erheblicher Intrusionskräfte. Bei einer Supraposition von 0,25 mm wurde für die meisten Positioner ein Kraftniveau von ungefähr $10 \mathrm{~N}$ ermittelt; eine Ausnahme bildete der thermogeformte Positioner mit einer Intrusionskraft von $32 \mathrm{~N}$. Bei einer Supraposition von 0,5 mm lagen die meisten Kräfte bei etwa 20 N. Die Ausnahme bildete wieder der thermogeformte Positioner, der Kräfte außerhalb des Messbereichs des Sensors ergab. Bei $1 \mathrm{~mm}$ Supraposition erreichten die Kraftniveaus $30 \mathrm{~N}$. Odontosil sh 60 und Scheu-EVA D80 lagen jedoch außerhalb des Messbereichs.

Die Infraposition des Eckzahnes führte nicht zur Erzeugung eindeutiger Extrusionskräfte, sondern eher zu einer Minderung des Niveaus der Intrusionskräfte gegenüber dem Modell mit dem Eckzahn in Supraposition. Bei 0,25 mm Infraposition zeigten alle Positioner Intrusionskräfte von etwa 2,5 N, mit Ausnahme des thermogeformten Positioners, der höhere Kräfte erreichte. Selbst bei mp3 wurde nur für die beiden Silikon-Positioner eine leichte Extrusionskraft von 0,3 $\mathrm{N}$ erfasst. Bei 0,5 mm Infraposition waren die Intrusionskräfte an dem Eckzahn reduziert und bei $1 \mathrm{~mm}$ Infraposition konnten fast keine Kräfte gemessen werden. Bei keinem der Niveaus der Infraposition kam es zur Erzeugung von Extrusionskräften.

In Verbindung mit der Mesiorotation des Eckzahns kam es zur Erzeugung eines Derotationsmoments. Bei Rotationen von $5^{\circ}$ erzeugten die meisten Positioner Derotationsmomente von etwa $1,5 \mathrm{Nmm}$. Bei einer Initialrotation von $10^{\circ}$ stiegen die Drehmomente beträchtlich an, und die meisten entgegenwirkenden Drehmomente wurden bei um die $5 \mathrm{Nmm}$ erfasst. Bei $20^{\circ}$ Rotation stiegen die durch die meisten Positioner einwirkenden Drehmomente auf ein Niveau um die $15 \mathrm{Nmm}$, während beide EVA-Positioner außerhalb des Messbereichs des Kraftsensors lagen. Selbst bei mp3 und mp4 wirkte von den meisten Positionern noch ein Derotationsmoment auf den Eckzahn ein.

Die Kippung erzeugte Aufrichtungsmomente von etwa 5-10 Nmm. Ähnlich wie bei der vertikalen Kraftabgabe wurden für den Positioner Scheu-EVA sh 80 die höchsten Aufrichtungsmomente mit einem Durchschnittswert von 11,9 Nmm erfasst. Polyurethan sh 70 hingegen zeigte mit 5,99 Nmm nur noch die Hälfte der Größenordnung des vorgenannten Aufrichtungsmoments.

Was den Torque anbelangte, waren die erreichten Drehmomente nicht voraussagbar. Während die meisten Positioner 


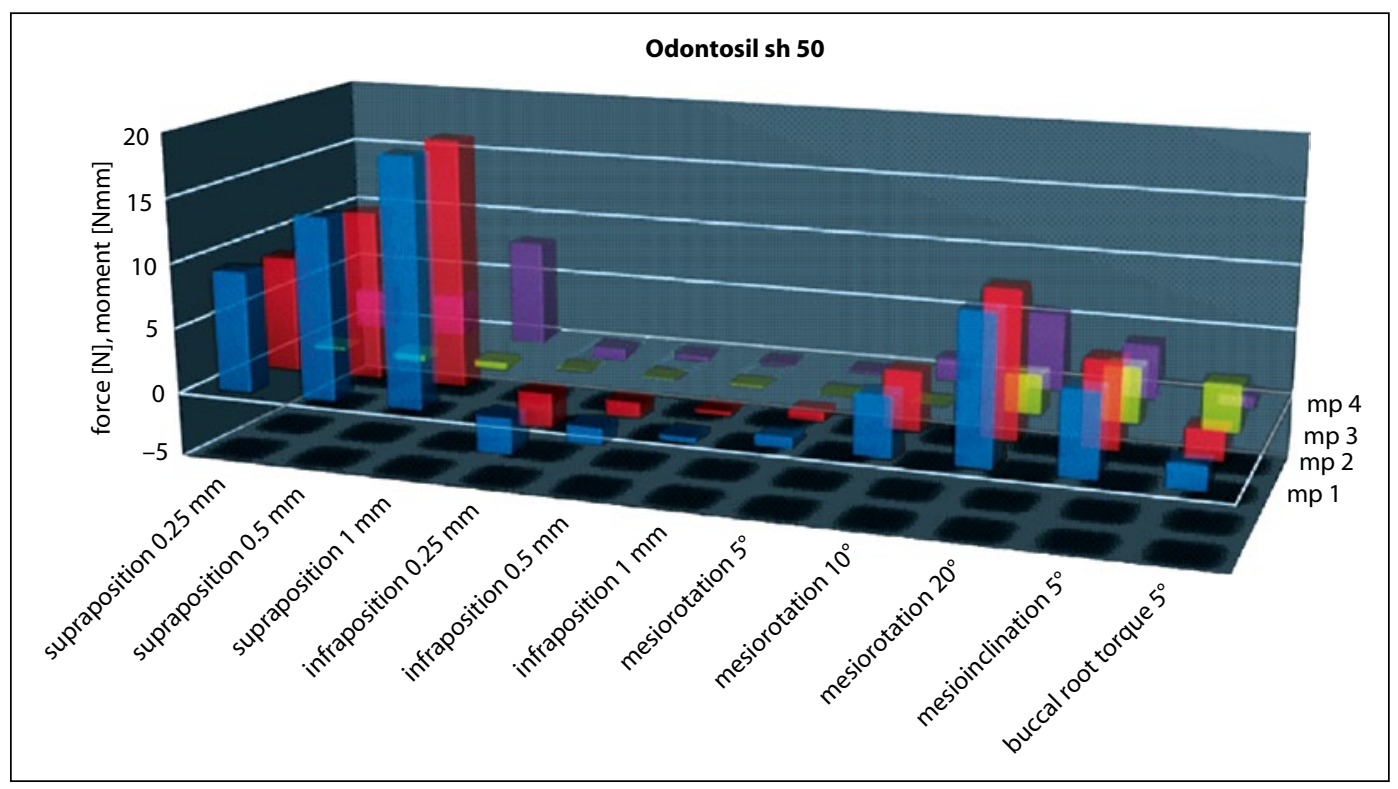

Figure 6. Example of force or moment transduction by an exemplary positioner (Odontosil sh 50) including all 11 malpositions and all measurement points. Considerable forces and moments in conjunction with teeth in supraposition, rotations $\geq 10^{\circ}$, and mesioinclination were noted. Positive values indicate beneficial therapeutic effects. (Jaw closing movement was defined by 4 mesurement points [mp]. Mp1: jaw closure by $0.5 \mathrm{~mm}, \mathrm{mp} 2$ : holding the closed position for $3 \mathrm{~s}, \mathrm{mp} 3$ : jaw opening by $1.5 \mathrm{~mm}, \mathrm{mp} 4$ : neutral position)

Abbildung 6. Beispiel für die Kraft- oder Momentübertragung für einen beispielhaften Positioner (Odontosil sh 50) unter Einbeziehung aller 11 Fehlstellungen und aller Messpunkte. Erhebliche Kräfte oder Drehmomente wurden erfasst bei Zähnen in Supraposition, Rotationen von $10^{\circ}$ oder höher sowie für die Mesioinklination. Positive Werte geben nützliche therapeutische Effekte an. (Für die Kieferbewegung waren vier Messpunkte (mp) definiert. Mp1 : Kieferschluss um 0,5 mm, mp2:Verharren in geschlossener Position für 3 Sekunden, $\mathrm{mp3}$ : Öffnung um 1,5 mm, mp4: neutrale Stellung)

Mesial rotation of the canine was associated with a derotational moment. At rotations of $5^{\circ}$, most postitioners led to derotational moments of approximately $1.5 \mathrm{Nmm}$. At $10^{\circ}$ of initial rotation, the moments increased considerably, and most counteracting moments occurred at about $5 \mathrm{Nmm}$. At $20^{\circ}$ of rotation, the moments exerted by most positioners increased to levels around $15 \mathrm{Nmm}$, with both EVA positioners outside the force sensor's measurement range. Even at $\mathrm{mp} 3$ and $\mathrm{mp} 4$, most positioners continued to exert a derotational moment on the canine.

Tipping led to uprighting moments of approximately 5-10 $\mathrm{Nmm}$. Similar to the delivery of vertical forces, the Scheu-EVA sh 80 positioner led to the highest uprighting moments with a mean of $11.9 \mathrm{Nmm}$. Polyurethane sh 70 achieved with $5.9 \mathrm{Nmm}$ only half the magnitude of the previously mentioned uprighting moment.

The moments achieved in conjunction with torque were unpredictable. Whereas most positioners did evoke labial crown torque, others (Scheu-EVA sh 80 , Silicone sh 50) led to palatal crown torque. With the exception of the Scheu-EVA sh 80 positioner, all measured moments were minor (range $-1.8 \mathrm{Nmm}$ to $+3.1 \mathrm{Nmm}$ ).

\section{Discussion}

A positioner's function can be evaluated via a clinical or in vitro approach. Although a clinical investigation would enable einen labialen Kronentorque erzeugten, erzeugten andere (Scheu-EVA sh 80, Silicone sh 50) einen palatalen Kronentorque. Mit Ausnahme des Positioners Scheu-EVA sh 80 hatten alle gemessenen Drehmomente einen geringen Wert $(-1,8$ bis $+3,1$ $\mathrm{Nmm}$ ).

\section{Diskussion}

Die Bewertung der Funktion eines Positioners kann grundsätzlich mit einem klinischen Ansatz oder in vitro erfolgen. Während eine klinische Studie die Bewertung der Wirksamkeit der Apparatur erlauben würde, liefert ein In-vitro-System Informationen über die möglichen Kraftsysteme, die auf die Zähne einwirken. Letzteres war das Ziel der vorliegenden Studie.

Da viele elastomere Materialien mit steigenden Temperaturen weicher werden, wurde in Übereinstimmung mit früheren Studien $[1,15,21]$ eine Versuchstemperatur von $36^{\circ} \mathrm{C}$ gewählt. Aus klinischen Studien $[12,14,20,22]$ und In-vitro-Versuchen $[1,15]$ ist ein erheblicher Einfluss der Kronenform auf die Wirksamkeit eines Positioners belegt. Der Eckzahn wurde gewählt, da seine Form in etwa zwischen der eines Schneidezahns und eines Prämolaren liegt. Die Berechnung der Bisskurve zeigte innerhalb des gemessenen Bereichs nur sehr geringe sagittale Abweichungen von einer rein vertikalen Bewegung (Abbildung 7) und ist daher für die zukünftige Forschung möglicherweise vernachlässigbar. Es wurde keine Schmierung verwendet, da der Pilotversuch kei- 


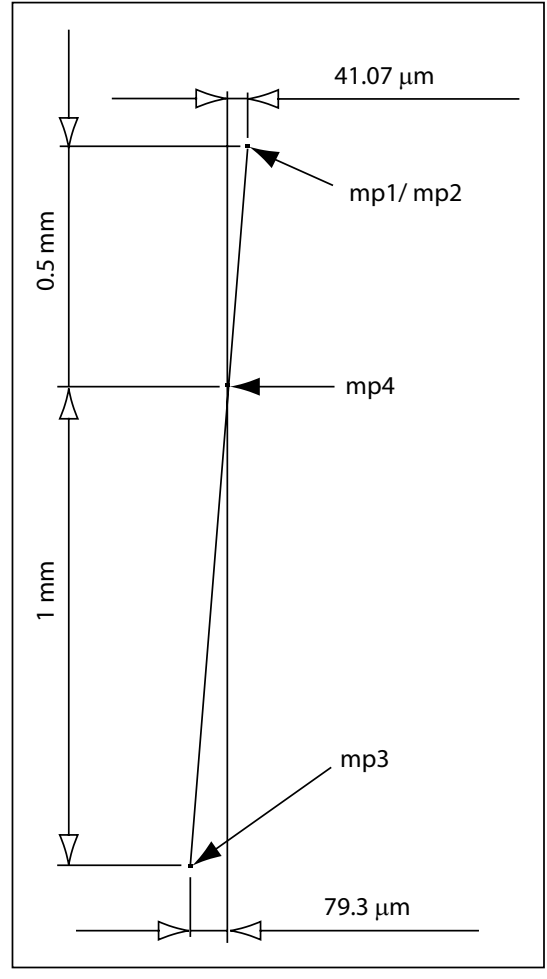

Figure 7. Calculation of mandibular movement in the positioner setup resulted in minor sagittal variations of $41 \mu \mathrm{m}$ for $0.5 \mathrm{~mm}$ of closing ( $\mathrm{mp} 1$, $\mathrm{mp} 2$ ) and $79 \mu \mathrm{m}$ for $\mathrm{mm}$ of opening (mp3)

Abbildung 7. Die Berechnung der Unterkieferbewegung im Positioner-Versuchsaufbau ergab lediglich geringfügige sagittale Abweichungen von $41 \mu \mathrm{m}$ für eine Bissschließung um $0,5 \mathrm{~mm}$ (mp1, mp2) und von $79 \mu \mathrm{m}$ für eine Bissöffnung um 1 mm (mp3)

appliance efficacy to be assessed, an in vitro design can provide information about the possible force systems applied to the teeth, which was the goal of this investigation.

As many elastomeric materials become softer as temperatures rises, an experimental temperature of $36^{\circ} \mathrm{C}$ was selected, which is in line with previous studies $[1,15,21]$. The crown form is known to have a significant influence on the effectiveness of a positioner in clinical studies $[12,14,20,22]$ as well as in vitro research $[1,15]$. For this study, it was decided to use the canine as it has a shape intermediate between that of the incisor and bicuspid. Calculation of the bite curve revealed very minor sagittal deviations from a pure vertical movement within the range measured (Figure 7) and can be neglected in future research. No lubrication was used since the pilot test did not show suction to play a significant role. However, it was noted that the transmitted forces reduced during the first 20 min of the measuring cycles remained almost stable thereafter. This effect had not been reported in earlier studies. Thus, it must have been due to an adaptation of the material, due to wear or more likely due to the elastic materials' flowability. These flowing characteristics may also be responsible for the force decay between $\mathrm{mp} 1$ and $\mathrm{mp} 2$, which was most pronounced in the EVA positioners. As the patient wears the positioner for many hours a day over several weeks, the initial $20 \mathrm{~min}$ in the evaluation protocol were excluded.

It is difficult to interpret the findings of our investigation in terms of the positioner's clinical effectiveness. It is important to remember that when doing biting exercises, the patient will not stop closing at a certain distance as the robotic device did, but rather at a certain and probably highly individual level of discom- nen Hinweis auf eine wesentliche Rolle von Saugkräften ergeben hatte. Es stellte sich jedoch heraus, dass die übertragenen Kräfte in den ersten 20 Minuten der Messzyklen zurückgingen und dann fast stabil blieben. Dies wurde aus vorherigen Studien nicht berichtet und war wahrscheinlich eine Folge der Anpassung der elastischen Materialien, entweder verschleißbedingt oder, was wahrscheinlicher ist, aufgrund der Fließfähigkeit. Diese Fließeigenschaften sind möglicherweise auch für den Kraftverfall zwischen mp1 und mp2 verantwortlich, der bei den EVA-Positionern am stärksten ausgeprägt war. Da der Positioner in der klinischen Anwendung über mehrere Wochen hinweg viele Stunden am Tag getragen wird, wurden die ersten 20 Minuten aus dem Auswertungsprotokoll herausgenommen.

Es ist schwierig, von den Ergebnissen der vorliegenden Studie auf die klinische Wirksamkeit des Positioners zu schließen. Es ist zu bedenken, dass der Patient bei der Ausführung von Beißübungen die Bewegungen für den Kieferschluss nicht bei einer bestimmten Entfernung anhält wie die Roboter-Apparatur, sondern an einem ganz bestimmten und sehr wahrscheinlich individuellen Punkt, der mit den von ihm verspürten Beschwerden sowie seiner Muskelaktivität zusammenhängt. In beiden Fällen würde es zu einer Beschränkung exzessiver Krafteinwirkung kommen. Darüber hinaus ist nicht klar, ob die aktive Phase der Schließbewegung oder eher die passive Führung den größten Einfluss auf die Okklusion hat $[15,18]$. Die Compliance mit den Beißübungen hat einen Einfluss auf die Wirkung während des Tages, aber die während der Nacht im Schlaf auf den Positioner einwirkenden Kräfte sind weniger voraussehbar. Aus einer früheren Studie sind starke Variationen sowohl der Beißkräfte (3$80 \mathrm{~N}$ ) als auch der Anzahl der Beißzyklen (39-558) dokumentiert [22]. Daher haben die in der vorliegenden Studie gemessenen Kräfte und Drehmomente lediglich einen indikativen Charakter hinsichtlich der relativen Wirksamkeit eines Positioners bei einer spezifischen Zahnfehlstellung und sind nicht direkt auf alle klinischen Situationen übertragbar.

Die Ergebnisse aus der vorliegenden Untersuchung stehen im Einklang mit früheren In-vitro-Studien [1, 15, 17]. Allgemein lässt sich aus den Ergebnissen schließen, dass die Intrusion die am besten mit einem Positioner korrigierbare Zahnbewegung ist. Alle Materialien zeigten starke Intrusionskräfte zwischen 6 und $32 \mathrm{~N}$ für Suprapositionen von $0,25 \mathrm{~mm}$ und $1 \mathrm{~mm}$ bei $\mathrm{mp} 1$. Obwohl diese für eine kontinuierliche Krafteinwirkung übermäßig hoch wären $[9,16,19]$, könnten sie für die intermittierende Krafteinwirkung angemessen sein. Eine Extrusion hingegen war nicht möglich. Stattdessen wurde bei mp1 und mp2 ein Intrusionseffekt beobachtet. Dieser Effekt war für eine Infraposition von $1 \mathrm{~mm}$ gering; jedoch wurden bei Infrapositionen von $0,25 \mathrm{~mm}$ ziemlich hohe Intrusionskräfte von bis zu $11 \mathrm{~N}$ gemessen. Bei 0,25 $\mathrm{mm}$ ist das nicht überraschend, da der Schlussbiss auf 0,5 mm eingestellt war; es ist deswegen $\mathrm{zu}$ erwarten, dass eine Intrusionskraft auf den Zahn einwirkt. Die für die Infraposition von $1 \mathrm{~mm}$ gemessenen geringen Intrusionskräfte waren möglicherweise die Folge einer Kompression des elastischen Materials an den Nachbarzähnen, die zu einer elastischen und möglicherweise plastischen Verformung des Positioners ge- 
fort or muscle activity. Either of these factors would limit excessive forces. Moreover, it is not clear whether it is the active phase on bite closure or the more passive guidance which exerts the greatest effect on occlusion $[15,18]$. Compliance with the biting exercises influences the positioner effect during the day, but its effect during sleep at night is less predictable. Strong variations in biting forces $(3-80 \mathrm{~N})$ and the number of biting cycles (39-558) were documented in an earlier study [22]. The forces and moments measured in our study are, therefore, only indicative of the relative efficacy of a positioner on a specific tooth malposition and cannot be simply extrapolated to all clinical situations.

The results from the present investigation are in line with those from earlier in vitro studies $[1,15,17]$. Generally speaking, our results provide evidence that intrusion is the tooth movement that best corrected with a positioner. All materials showed high intrusive forces between 6 and $32 \mathrm{~N}$ for suprapositions of $0.25 \mathrm{~mm}$ and $1 \mathrm{~mm}$ at $\mathrm{mp} 1$. Although these would be excessively high for continuous force application $[9,16,19]$, they could be appropriate for intermittent forces. Extrusion on the other hand was not possible; instead, an intrusive effect was observed at $\mathrm{mp} 1$ and $\mathrm{mp} 2$. The effect was small in conjunction with an infraposition of $1 \mathrm{~mm}$; however, rather high intrusive forces of up to $11 \mathrm{~N}$ with infrapositions of $0.25 \mathrm{~mm}$ were noted. At $0.25 \mathrm{~mm}$ this is not surprising, as bite closure was set at 0.5 $\mathrm{mm}$, so the tooth should be affected by intrusive force. The small, intrusive forces measured in conjunction with the $1 \mathrm{~mm}$ infraposition was probably caused by compression of the elastic material at the adjacent teeth, leading to elastic and maybe even plastic deformation of the positioner. A suction effect was not observed at mp3 or mp4; thus, the active extrusion of teeth does not seem possible with a positioner appliance, which is in line with previously published evidence $[1,15]$. However, as stronger intrusive forces will act on the adjacent teeth, a leveling effect may still become clinically apparent.

The application of moments for rotation, tip, and torque has been controversial, as it depends on the direction of the moment and the severity of the malposition. Rotations of $10^{\circ}$ and $20^{\circ}$ induced clinically advantageous moments at $\mathrm{mp} 1$, and for rotations of $20^{\circ}$, even at $\mathrm{mp} 3$ and $\mathrm{mp} 4$ simulating a resting position. In accordance with the literature $[1,15]$, our results with small rotations of $5^{\circ}$ indicate that minor rotations will probably not be corrected by wearing a positioner. For a $5^{\circ}$ mesial tip of a canine, the positioner displayed an adequate ability to correct the tooth position with moments between 6 and $12 \mathrm{Nmm}$. The forces applied by the positioner to a canine with buccal root torque, however, were inconsistent with moments acting in opposite directions. This observation is reported in two clinical reports [18, $22]$, but is contradicted by others $[1,15]$, showing a corrective capacity of approximately $45 \%$.

Considering the different materials tested, we noted generally stronger forces or moments in conjunction with materials featuring higher shore hardness as expected. The highest forces or moments appeared with the vacuum-thermoformed Scheu EVA positioner with a shore hardness of 80 , the lowest with the silicone positioner having a shore hardness of 50 . The relative führt haben könnten. Ein Saugeffekt bei mp3 oder mp4 konnte nicht beobachtet werden. Deswegen scheint eine aktive Extrusion der Zähne mit einer Positioner-Apparatur nicht möglich zu sein, was im Einklang mit der früheren Literatur steht $[1,15]$. Da jedoch auf die Nachbarzähne stärkere Intrusionskräfte einwirken werden, könnte in der klinischen Anwendung immer noch ein Nivellierungseffekt beobachtet werden.

Die Applikation von Drehmomenten für die Rotation, Kippung und Torque zeigte aufgrund ihrer Abhängigkeit von der Richtung des Drehmoments sowie von dem Schweregrad der Fehlstellung widersprüchliche Ergebnisse. Rotationen von $10^{\circ}$ und $20^{\circ}$ ergaben bei mp 1 und für Rotationen von $20^{\circ}$ selbst bei mp3 und mp4 (Simulation der Ruhestellung) klinisch nützliche Drehmomente. Gemäß der früheren Literatur $[1,15]$ verweisen die Ergebnisse für geringfügige Rotationen von $5^{\circ}$ darauf, dass geringgradige Rotationen mit einem Positioner wahrscheinlich nicht korrigierbar sind. Bei der Mesialkippung des Eckzahns um $5^{\circ}$ zeigte der Positioner eine hinreichende Fähigkeit zur Korrektur der Zahnstellung mit Drehmomenten zwischen 6 und $12 \mathrm{Nmm}$. Die von dem Positioner auf einen Eckzahn mit bukkalem Wurzeltorque einwirkenden Kräfte waren jedoch mit den in andere Richtungen wirkenden Drehmomenten inkonsistent. Dies deckt sich mit zwei klinischen Berichten [18, 22], widerspricht aber anderen $[1,15]$, aus denen eine Korrekturfähigkeit von etwa $45 \%$ hervorgeht.

Unter Berücksichtigung der verschiedenen getesteten Materialien ergaben sich die stärkeren Kräfte oder Drehmomente in der Regel bei den Materialien mit einer höheren Shore-Härte. Dies war zu erwarten. Die höchsten Kräfte und Drehmomente wurden bei dem vakuum-thermogeformten Scheu EVA-Positioner mit einer Shore-Härte von 80 und die niedrigsten für den Silikon-Positioner mit einer Shore-Härte von 50 gemessen. Die relativen Unterschiede zwischen den beiden Positionern waren nicht für alle Fehlstellungen konsistent. Es ist möglich, dass die Shore-Härte auf unterschiedliche Fehlstellungen in einem unterschiedlichen Ausmaß einwirkt. Obwohl die Kraftabgabe durch härtere Positioner in klinischer Hinsicht wahrscheinlich effektiver ist, werden Positioner aus weicheren Materialien wahrscheinlich besser von den Patienten toleriert. Andere bei der Effektivität der Kraftübertragung zu berücksichtigende Materialeigenschaften sind die Reibung, das elastische Verhalten und schließlich die Materialdicke, die alle entsprechend den verschiedenen Herstellungsmethoden variieren können.

Die Ergebnisse unserer Studie verweisen darauf, dass der Positioner klinisch am wirksamsten ist bei der Steuerung der Konsolidierung der Okklusion durch Intrusions- und Kippbewegungen

\section{Schlussfolgerung}

- Die für die Herstellung der Positioner verwendeten Materialien hatten einen wesentlichen Einfluss auf die registrierten Kräfte und Drehmomente, wobei härtere Materialien eine bessere Fähigkeit zur Applikation größerer Kräfte und Drehmomente zeigten. 
differences between the two positioners were not consistent for all malpositions. Shore hardness may affect different malpositions to various degrees. Although clinically harder positioners probably apply more effective forces, patients will most likely prefer positioners made from softer material. Other material characteristics to be considered in the effectiveness of force transduction include friction, elastic behavior, and ultimately material thickness, all of which will vary due to different methods of fabrication.

The results from our study imply that the positioner is most clinically effective when guiding the settling of the occlusion via intrusion and tipping.

\section{Conclusion}

- The materials used in the fabrication of the positioners had a major influence on the forces and moments recorded, with harder materials revealing a greater capacity for applying larger forces or moments.

- An elastic positioner transmits intrusive forces to teeth easily. No extrusive forces were observed. The successful treatment of open bite cases already reported may be due to posterior intrusion and the guidance of tooth eruption rather than active extrusive forces.

- Advantageous rotational moments in conjunction with $10^{\circ}$ and $20^{\circ}$ of rotation were observed, whereas minor rotations of $5^{\circ}$ did not lead to significant moments.

- Beneficial uprighting moments were apparent at $5^{\circ}$ of mesial tip. - The application of torque was unreliable.

\section{Conflict of interest}

The corresponding author states that there are no conflicts of interest.

\section{References}

1. Bourauel C, Drescher D, Wolter H (1997) Können die Kraftsysteme von Positionern durch den zusätzlichen Einsatz von Attachments beeinflusst werden? Eine experimentelle Studie. Kieferorthop 11:183-190

2. Bowman SJ, Carano A (2002) Short-term, intensive use of the tooth positioner in case finishing. J Clin Orthod 36:216-219

3. Cottingham LL (1969) Gnathologic clear plastic positioner. Am J Orthod 55:2331

4. Craig RG, Godwin WC (1967) Physical properties of materials for custom-made mouth protectors. J Mich State Dent Assoc 49:34-40

5. Elsasser WA (1950) Some observations of the history and uses of the Kesling positioner. Am J Orthod 36:368-374

6. Kamada K, Tamura T, Hama Y et al (1984) Case reports on tooth positioners using LTV vinyl silicone rubber. J Nihon Univ Sch Dent 26:11-29

7. Kesling HD (1946) Coordinating the predetermined pattern and tooth positioner with conventional treatment. Am J Orthod Oral Surg 32:285-293

8. Kesling HD (1945) The philosophy of the tooth positioning appliance. Am J Orthod Oral Surg 31:297-304

9. Lee BW (1965) Relationship between tooth-movement rate and estimated pressure applied. J Dent Res 44:1053-1057

10. Moore DJ, Glaser ZR, Tabacco MJ, Linebaugh MG (1977) Evaluation of polymeric materials for maxillofacial prosthetics. J Prosthet Dent 38:319-326
- Ein elastischer Positioner kann leicht Intrusionskräfte auf die Zähne übertragen. Extrusionskräfte konnten nicht beobachtet werden. Die Berichte über eine erfolgreiche Behandlung von Fällen mit offenem Biss könnten auf eine posteriore Intrusion und eine Steuerung des Zahndurchbruchs anstatt auf das aktive Einwirken von Extrusionskräften zurückzuführen sein.

- Nützliche Rotationsmomente wurden für eine Rotation von $10^{\circ}$ und $20^{\circ}$ erfasst, während geringfügigere Rotationen von $5^{\circ}$ keine signifikanten Drehmomente erzeugten.

- Bei einer Mesialkippung von $5^{\circ}$ wurden nützliche Aufrichtungsmomente ermittelt.

- Die Applikation von Torque war unzuverlässig.

\section{Interessenkonflikt}

Der korrespondierende Autor gibt an, dass kein Interessenkonflikt besteht.

11. Nishiyama M, Kamada K, Horiuchi S (1977) A new construction of tooth positioner with LTV vinyl silicone rubber. J Nihon Univ Sch Dent 19:93-102

12. Park Y, Hartsfield JK, Katona TR, Roberts WE (2008) Tooth positioner effects on occlusal contacts and treatment outcomes. Angle Orthod 78(6):1050-1056

13. Permann I, Bantleon HP, Muchitsch AP, Droschl H (1989) Der Positioner - eine Möglichkeit zur Feineinstellung der Okklusion, objektiviert durch Kondymetermessungen der Diskrepanz zwischen IKP und RKP. Fortschr Kieferorthop 50:530-539

14. Posselt U (1956) Hinge opening axis of the mandible. Acta Odontol Scand 57(14):49-63

15. Prestel B, Drescher D, Bourauel C (1995) Kräfte und Drehmomente beim Einsatz von Positionern. Kieferorthopädie 9:117-120

16. Reitan K (1957) Some factors determining the evaluation of forces in orthodontics. Am J Orthod 43:32-45

17. Rost D, Schwarze CW, Hilgers RD (1995) Die Kraftabgabe von Positionern bei unter-schiedlicher Schneidezahnprotrusion. Eine In-vitro-Untersuchung. Fortschr Kieferorthop 56:104-109

18. Sander FG, Fröhls H (1989) Qualitative and quantitative Bestimmung des settling-Effekts durch die Positioner-Therapie. Inf Orthod Kieferorthop 21:311-318

19. Smith R, Storey E (1952) The importance of force in orthodontics. Aust J Dent 56:291-304

20. Vorhies JM (1960) Short, intensive use of tooth positioners and an appraisal of the results. Angle Orthod 30:248-54

21. Warunek SP, Sorensen SE, Cunat JJ, Green LJ (1989) Physical and mechanical properties of elastomers in orthodontic positioners. Am J Orthod Dentofacial Orthop 95:388-400

22. Wells NE (1970) Application of the positioner appliance in the orthodontic treatment. Am J Orthod 58:351-366

23. Wichelhaus A, Hüffmeier S, Sander FG (2003) Dynamic functional force measurements on an anterior bite plane during the night. J Orofac Orthop 64:417-425

\section{Correspondence address}

Dr. Dr. Lorenz Brauchli

Department of Orthodontics and Pedodontics

University of Basel

Hebelstrasse 3

4056 Basel

Switzerland

e-mail: lorenz.brauchli@unibas.ch 